\title{
$\overline{A V}$ A review of non-destructive techniques applied for measuring quality of oil palm fresh fruit bunches
}

Chen Yee Beinga, ${ }^{*}$, Norhashila Hashima,b, Bernard Maringgala, Mohd Hafizz Wondic

${ }^{a}$ Department of Biological and Agricultural Engineering, Faculty of Engineering, Universiti Putra Malaysia, 43400 Serdang, Selangor, Malaysia. ${ }^{b}$ SMART Farming Technology Research Centre (SFTRC), Faculty of Engineering, Universiti Putra Malaysia, 43400 Serdang, Selangor, Malaysia. 'Department of Process and Food Engineering, Faculty of Engineering, Universiti Putra Malaysia, 43400 Serdang, Selangor, Malaysia.

\section{ARTICLE HISTORY}

Received: 2 March 2020

Received in revised form: 9 March 2020

Accepted: 10 March 2020

Available Online: 12 March 2020

\section{Keywords}

Oil palm

Fresh fruit bunches

Non-destructive

Ripeness

Quality

\section{Abstract}

The quality of oil palm fruits is evaluated through several characteristics such as the ripeness level, oil content, and free fatty acid. Besides human visual assessment and destructive techniques, another alternative method that provides quality assessment on oil palm fresh fruits bunches (FFB) is through the application of non-destructive techniques. A few of the non-destructive techniques are covered in this review such as machine vision system, visible or near infrared spectroscopy, image processing using relative entropy, fluorescence technique, Kinect camera, and optical sensor system. The main quality parameter that is being evaluated is the ripeness level of the FFB because the maturity of FFB has a direct impact on the quality of the extracted oil that will eventually affect the economic value of palm oil.

\section{Introduction}

Elaeis guineensis (subsps. Nigrescens) is known to be a commercial and popular oil palm species that is grown in Malaysia, which consists of three varieties which are tenera, dura, and pisifera (Hazir et al., 2012). According to Kushairi et al. (2019), Malaysia produced 17.16 tonnes oil palm fresh fruit bunches (FFB) every hectare in 2018. Malaysia's export earnings of palm oil and oil palm products were RM65.12 billion in that same year.

The quality of oil palm fruits is evaluated through several characteristics. Colour is the main characteristic to detect the ripeness of a fruit. Human visual perception is often used to differentiate colours but the outcomes may be varied and inconsistent due to physical and psychological state of humans (Makky, 2016). Furthermore, the quality of the fruit can also be checked by the amount of oil content as well as free fatty acid that significantly affect the quality of palm oil produced. Assessment on oil content and free fatty acid is normally via chemical analysis at laboratory which is costly and time consuming as well as destructive to the samples (Makky \& Soni, 2014).

Besides human visual assessment and destructive techniques, another alternative method that provides quality assessment on oil palm FFB is through the application of nondestructive techniques. Non-destructive techniques are proven to be reliable and efficient using advanced technologies with data handling and processing. Harun et al. (2013) stated that numerous automated fruit grading systems were proposed and their functionalities were tested in the past few years. A few of the non-destructive techniques are covered in this mini review such as machine vision system, visible or near infrared spectroscopy, image processing using relative entropy, fluorescence technique, Kinect camera and optical sensor system.

\section{Physiology and quality of oil palm}

Harvesters in oil palm sector are required to follow grading standard guidelines in order to avoid misclassification of oil palm ripeness. A summary of the grading standards established by Malaysian Palm Oil Board (MPOB), Sime Darby Palm Oil Mill and other experienced FFB mill graders are represented in Table 1 (Harun et al., 2013; Hazir, Shariff, \& Amiruddin, 2012). The first grading method is to identify the total number of empty sockets on the bunch and the colour of mesocarp. Mesocarp with yellow colour is considered as unripe whereas orange colour is categorized as ripe. Another method is to determine the FFB ripeness by basing on the number of detached fruitlets. The oil palm FFB is classified as ripe when the fruits detached from the bunch are between 10 to $50 \%$. Different ripeness level of FFB has different surface colour and condition as depicted in Figure 1.
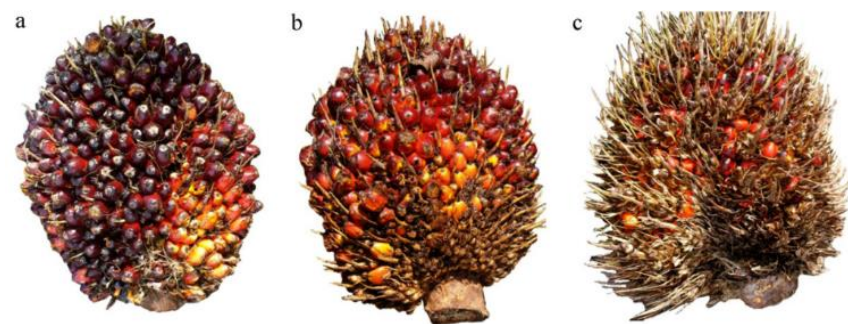

Figure 1. Oil palm FFB surface colour and condition in different categories or ripeness; (a) under-ripe; (b) ripe; and (c) over-ripe (Hazir, Shariff, \& Amiruddin, 2012)

According to Makky and Soni (2014), the level of oil palm fruits or bunches ripeness can be determined by computing the ratio of carotenoids to chlorophyll pigments in fruit skin or vice versa. In raw fruits, the presence of chlorophyll is the highest whereas the carotenoid is the lowest. As the ripening process 
Table 1. Grading standard used to determine ripeness of oil palm FFB (Harun et al., 2013)

\begin{tabular}{|c|c|c|c|c|}
\hline \multirow[t]{2}{*}{ Grading Method } & \multirow{2}{*}{$\begin{array}{l}\text { Total Number of Empty } \\
\text { Fruitlet Sockets }\end{array}$} & \multicolumn{3}{|c|}{ Mesocarp Colour } \\
\hline & & Yellow & Orange/Yellowish & Orange \\
\hline \multirow{3}{*}{$\begin{array}{l}\text { Number of loose fruit } \\
\text { sockets on the bunch }\end{array}$} & 0 & Unripe & Unripe & Ripe \\
\hline & $0-10$ & Unripe & Under-ripe & Ripe \\
\hline & $>10$ & Unripe & Ripe & Ripe \\
\hline \multirow{3}{*}{$\begin{array}{l}\text { Number of loose fruits on } \\
\text { the ground }\end{array}$} & Ripe & \multirow{3}{*}{\multicolumn{3}{|c|}{$\begin{array}{l}\text { Fruits detached from bunch: } 10 \%-50 \% \\
\text { Fruits detached from bunch: } 50 \%-90 \% \\
\text { Fruits detached from bunch: } 1-9\end{array}$}} \\
\hline & Over-ripe & & & \\
\hline & Under-ripe & & & \\
\hline
\end{tabular}

occurs, chlorophyll decreases while carotenoid increases. This biochemical reaction causes the change in surface colour of the mesocarp. The location of mesocarp in the fruitlet is shown in Figure 2. The colour change of the fruitlets begins from black to purple, and then orange to red. As for the whole bunch, the ripening process initiates from apical part to basal part.

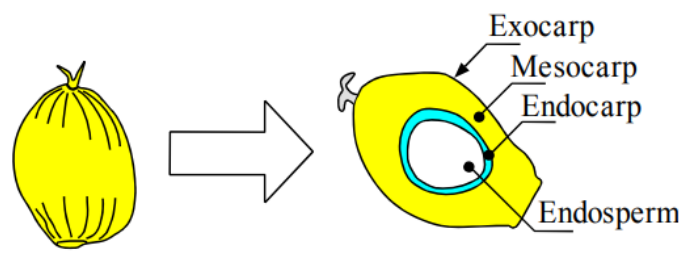

Fruitlet

Figure 2. Cross-sectional view of oil palm fruitlet (Harun et al., 2013)

Harun et al. (2013) stated that chemical analysis on ripe and unripe fruitlets was performed at Food Technology Department, General Industrial Technology Center, Nagano Prefecture, Japan. The result of the analysis on chemical contents is shown in Figure 3. Ripe fruitlet contains the highest amount of lipid or oil with $58.3 \%$ whereas unripe fruitlet has the highest moisture content of $80.1 \%$. Whereas, $17.4 \%$ in ripe fruitlet and $14 \%$ in unripe fruitlet are actually the fiber component of the fruit.

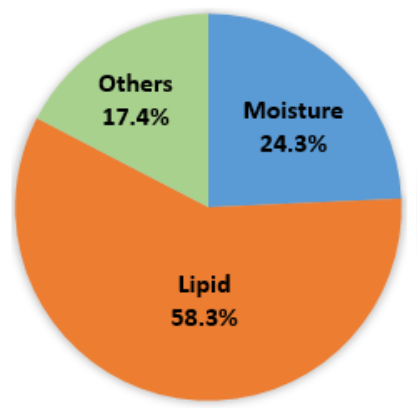

(a)

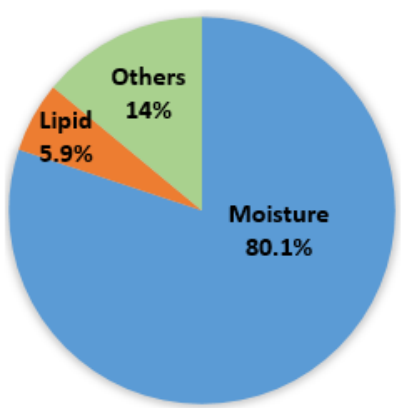

(b)
Figure 3. Chemical analysis on oil palm fruitlet; (a) ripe; and (b) unripe (Harun et al., 2013)

Free fatty acid (FFA) level influences the oil palm quality. A higher level of FFA leads to the increase in chemical processing which eventually contributes to a rise in the production cost. Palm oil that contains high amount of FFA is rated as poor quality (Makky \& Soni, 2014). In addition, oil extraction rate (OER) is taken into consideration as OER acts as a benchmark to evaluate the performance of plantation and mill. This evaluation is done by assessing the level of oil comprehends for every hectare of land under cultivation. OER is calculated by dividing the weight of physically recovered oil with the weight of processed FFB. The quality is optimal when OER is higher than $21 \%$ and FFA is less than $5 \%$ (Hazir et al., 2012). The FFB that is harvested at its peak of ripeness is able to maximize the OER (Kassim et al., 2014).
Generally, oil palm FFB quality can be assessed by ripeness level through measuring surface colour, oil extraction rate or oil content, and the amount of free fatty acid. The quality of FFB needs to be checked properly in order to produce high quality palm oil while reducing excess operation costs.

\section{Non-destructive techniques for measuring quality of oil palm}

Table 2 shows the application of various non-destructive techniques in evaluating the quality of oil palm fresh fruit bunches (FFB). VIS/NIR spectroscopy, machine vision inspection system, relative entropy-based image processing, fluorescence technique, Kinect camera and portable four-band optical sensor system are the non-destructive techniques involved for measuring quality of oil palm FFB.

\subsection{Machine vision inspection system}

According to Makky (2016), this study applied machine vision inspection system to detect fruit ripeness. This system was developed with a chamber, a camera (Finepix J27, Fuji Film, Japan) and a computer as shown in Figure 4. Three categories of features were extracted from the captured FFB image, comprising of colour channels (red, green and blue), chromaticity data (hue, intensity and saturation) and normalization value of the colour channels. Discriminate analysis was performed on these categories of features to create canonical discriminant function for the classification of FFB ripeness. This system was able to classify $85 \%$ of the FFB samples accurately. Moreover, this system had the ability to produce free fatty acid and oil content modelling. The free fatty acid and oil content prediction models were created using multiple linear regression analysis. The result of oil content model was acceptable with a coefficient of determination $\left(R^{2}\right)$ of 0.931 and a standard error of prediction (SEP) of 0.821 whereas the performance of free fatty acid model was poor with SEP of 0.71 and $\mathrm{R}^{2}$ of 0.26 . The on-site operation was allowed due to light weight and good mobility of this system. However, machine vision inspection system uses batteries as power supply whereby recharging of this system is necessary after 6 hours.

\subsection{VIS/NIR spectroscopy}

VIS/NIR spectroscopy was developed to observe and measure the ripeness, free fatty acid and oil content via spectral reflectance analysis. This rapid and cost-effective technique facilitated continuous quality evaluation through visible and near infrared spectrum whereby the ratio of carotenoids to chlorophyll and internal properties of fruits were assessed. Ocean Optic USB2000+VIS-NIR series spectrometer, QR600-7VIS-NIR optical fiber reflection probes and HL-2000 tungsten halogen light sources (Ocean optics, USA) were employed in reflectance spectral measurements as shown in Figure 5 a. Ambient light was prevented from reaching the sensor by perpendicularly placing the reflectance probe to the surface of 


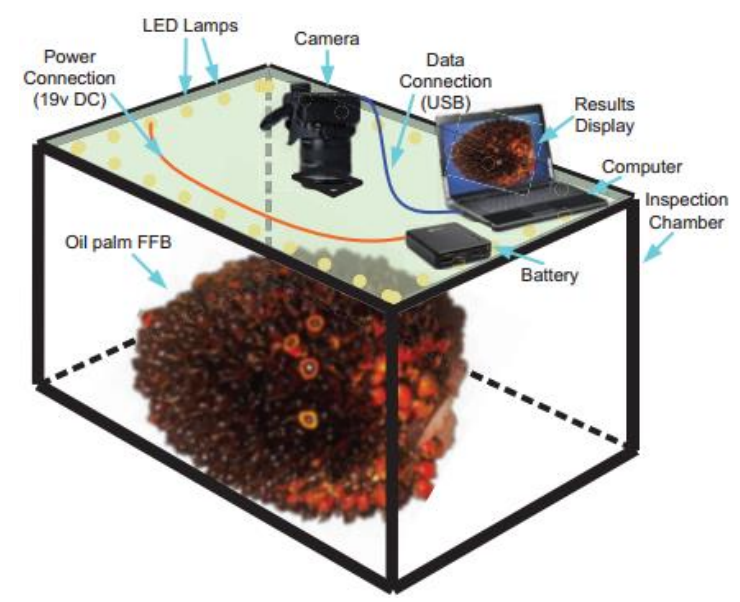

Figure 4. Components of machine vision inspection system (Makky, 2016)
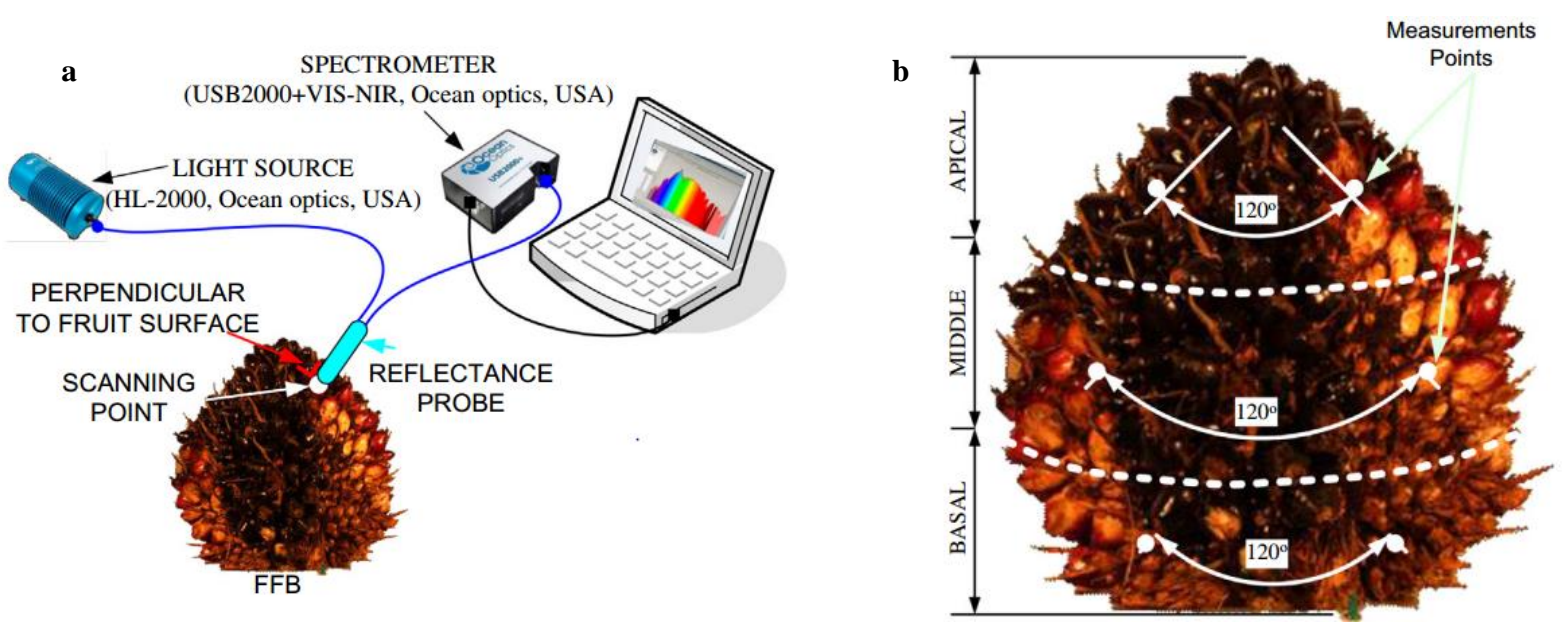

Figure 5. (a) Experimental set-up; and (b) Measurement position around equator for basal, middle, and apical parts of FFB (Makky \& Soni, 2014)

Table 2. Summaries of non-destructive measurements applied for quality evaluation of oil palm FFB

\begin{tabular}{|c|c|c|c|}
\hline Application & Quality parameters & Data / classification analysis & References \\
\hline \multirow[t]{3}{*}{ Machine vision inspection system } & Ripeness & Discriminate analysis & Makky (2016) \\
\hline & Oil content & Multiple linear regression analysis & \\
\hline & Free fatty acid & Multiple linear regression analysis & \\
\hline \multirow[t]{3}{*}{ VIS/NIR spectroscopy } & Ripeness & FS-MLR and PCA-MLP & Makky \& Soni (2014) \\
\hline & Oil content & FS-MLR and PCA-MLP & \\
\hline & Free fatty acid & FS-MLR and PCA-MLP & \\
\hline $\begin{array}{l}\text { Relative entropy-based image } \\
\text { processing }\end{array}$ & Ripeness & Relative entropy (KL distance) & $\begin{array}{l}\text { Taparugssanagorn et al. } \\
(2015)\end{array}$ \\
\hline Fluorescence technique & Ripeness & C\&RT & Hazir et al. (2012) \\
\hline \multirow[t]{2}{*}{ Kinect camera } & Volume & SVIS algorithm & Pamornnak et al. (2017) \\
\hline & Colour appearances & Non-linear regression technique & \\
\hline \multirow{2}{*}{$\begin{array}{l}\text { Portable four-band optical sensor } \\
\text { system }\end{array}$} & Ripeness & QDA & Saeed et al. (2012) \\
\hline & Ripeness & DA based on Mahalanobis distance & \\
\hline Backscattering imaging system & $\begin{array}{l}\text { Ripeness, color, } \\
\text { oil content }\end{array}$ & PCA, PLS, LDA, QDA & Mohd Ali et al. (2020) \\
\hline
\end{tabular}


the fruit. The measurements were obtained at nine different points with repetition of three times per point for each bunch of oil palm fruit (Figure 5b). From 96 FFB samples, a total of 2,592 reflectance spectra data were collected. The wavelength between $400 \mathrm{~nm}$ to $1000 \mathrm{~nm}$ was considered in order to ensure a high signal-to-noise ratio is obtained. PCA-MLP and FS-MLR were the two methods that were used to develop prediction models for ripeness, oil content and free fatty acid. Forward stepwise (FS) was used to achieve simpler model and eliminate collinear variables whereas PCA was employed to extract four principal components from spectral data. Makky and Soni (2014) concluded that the models created using FS-MLR method had a better performance because more predictor variables were employed.

\subsection{Relative entropy-based image processing}

Taparugssanagorn, Siwamogsatham and Pomalaza-Ráez (2015) proposed a simple non-destructive oil palm ripeness recognition using image processing with information theory. The images were taken in a natural light environment by a digital camera with similar specifications in any smart phone. The digital image pre-processing was carried out to enhance image characteristics such as brightness and contrast adjustment as well as deblurring. Figure 6 shows the procedure of the proposed oil palm classification technique. Three maturity levels were considered namely completely ripe, medium ripe, and unripe. The ripeness level of oil palm was predefined by standard scale images. The differences of the distributions of a testing image and standard scale images were computed in terms of relative entropy, also known as Kullback-Leibler distance (KL distance) using Matlab implementation. The determination of ripeness level was made based on the distance of standard scale image from the testing image. The image from the standard scale with minimum distance had the largest similarity to the testing image. The average performance of proposed approach was $96 \%$ accurate when tested with one hundred images of the Nigrescens-type oil palm FFB. Similar results were obtained from the mobile application developed in this study.

\subsection{Fluorescence technique}

A research was done at an oil palm plantation in peninsular Malaysia where a total of two hundred and ten oil palm FFB from three categories of under-ripe, ripe and over-ripe were scanned with a hand-held multi-parameter fluorescence sensor, Multiplex 3. Each sample was scanned ten times randomly. In this study, the parameter measured was the Blue-to-Red Fluorescence Ratio (BRR_FRF) obtained from blue-green (447 $\mathrm{nm})$ and far-red ( $685 \mathrm{~nm}$ ) emission signal by using ultraviolet (UV) light emitting diode as excitation light source. This research showed that BRR_FRF index was capable to provide a significant difference among the three maturity categories. Classification and Regression Tree (C\&RT) method was performed to separate oil palm FFB into their respective categories. This method resulted in an overall classification accuracy up to $90 \%$ (Hazir, Shariff, Amiruddin, et al., 2012).

\subsection{Kinect camera}

The grading of palm bunch was conducted using a Microsoft Kinect 2.0 (1920 x 1080 pixels for RGB and $512 \times 424$ pixels for point cloud and infrared images) that was installed $1 \mathrm{~m}$ above conveyor line for image acquisition module (Figure 7). Several features were obtained which are hue value from RGB images, volume index from point cloud and infrared intensity from

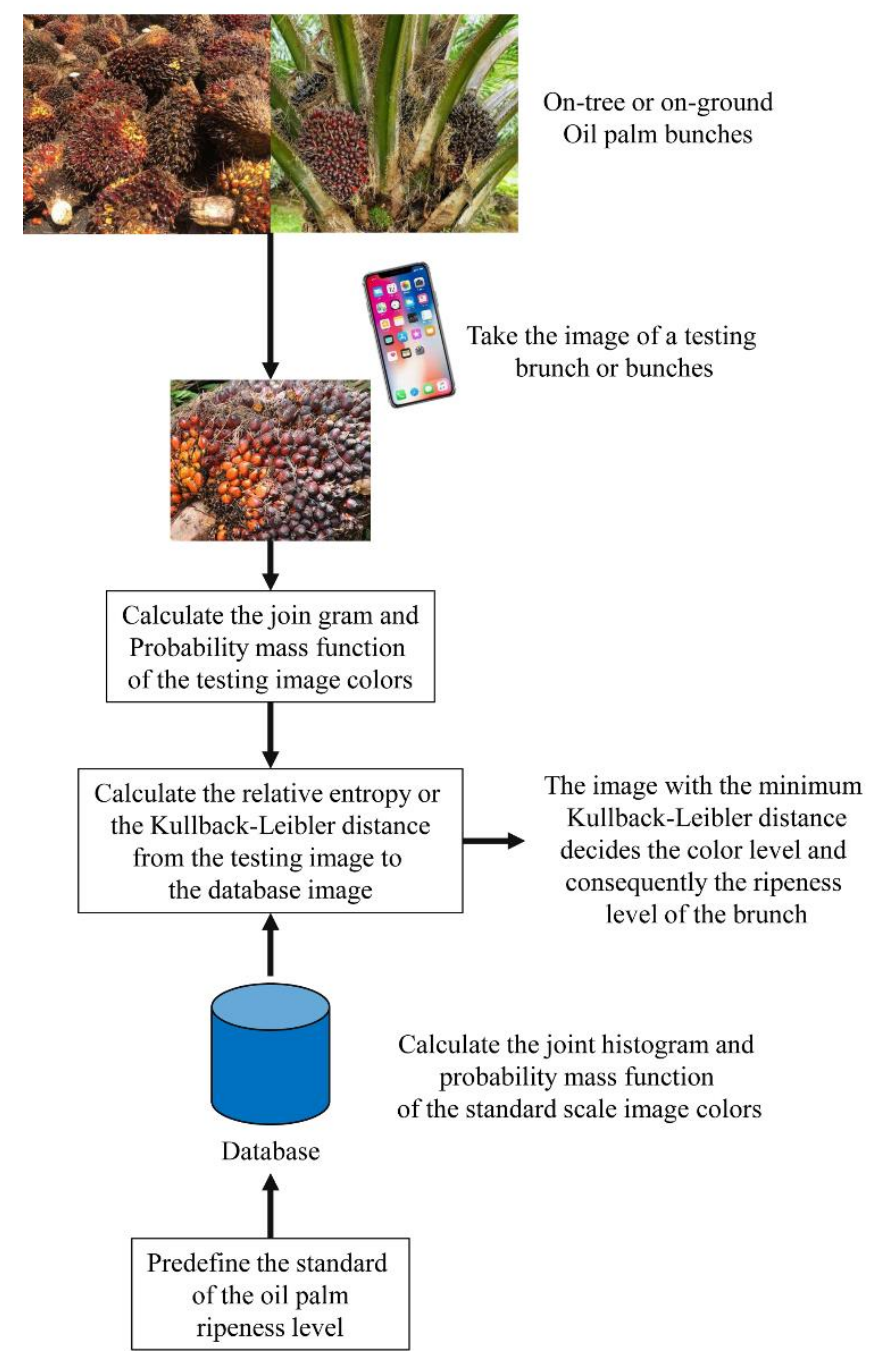

Figure 6. Block diagram of the proposed procedure (Taparugssanagorn et al., 2015)

infrared images. Two main algorithms for classification were developed in this study. An algorithm used to measure the relative volume index, known as simple volume integration scheme (SVIS) obtained $2.94 \%$ volume error for $9200 \mathrm{~cm}^{3}$ object. This algorithm achieved $\mathrm{R}^{2}$ of 0.911 for linear correlation between volume index values and bunch weight, thus SVIS was proven to be applicable in determining bunch weight. The function of another classification algorithm was to grade quality palm bunch into low, medium and high grade depending on its oil content. Pamornnak et al. (2017) reported that this system was able to grade 10 palm bunches per minute with a successful rate of $83 \%$.

\subsection{Portable four-band optical sensor system}

In a study conducted by Saeed et al. (2012), a hand-held four-band optical sensor system was utilized to acquire reflectance data from 120 fresh fruit bunches (Figure 8). The system comprised of four narrow-band (active optic) light sources and reflectance sensing elements of variable wavelengths which are $570 \mathrm{~nm}$ (visible), $670 \mathrm{~nm}$ (visible), 750 $\mathrm{nm}$ (red-edge) and $870 \mathrm{~nm}$ (near infrared). An epoxy lens type illuminator (Marubeni America Corporation, Santa Clara, CA 95054), assembled with 60 high efficiency aluminium gallium arsenide diode chips was applied for the four bands. These fresh fruit bunches were classified into unripe, ripe and overripe classes by using different classifiers. Discriminant analysis 

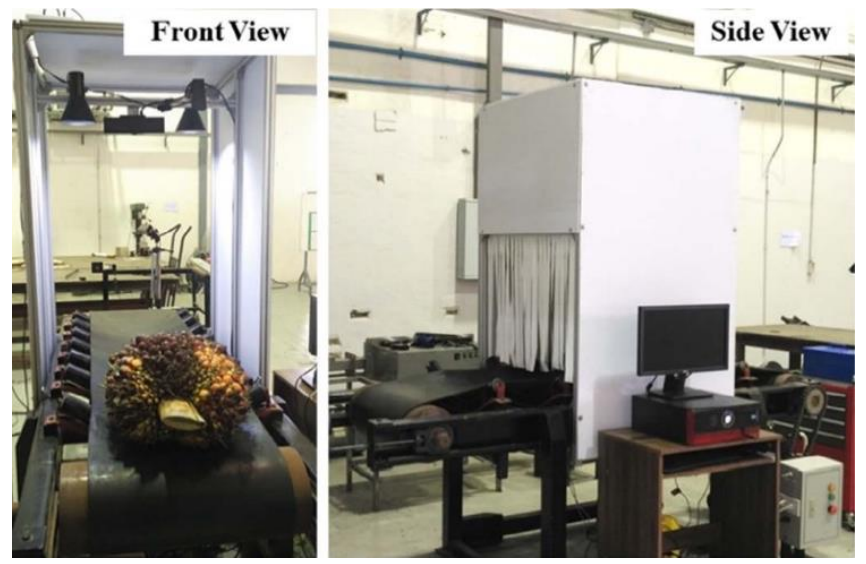

Figure 7. The complete system used for palm bunch grading (Pamornnak et al., 2017)

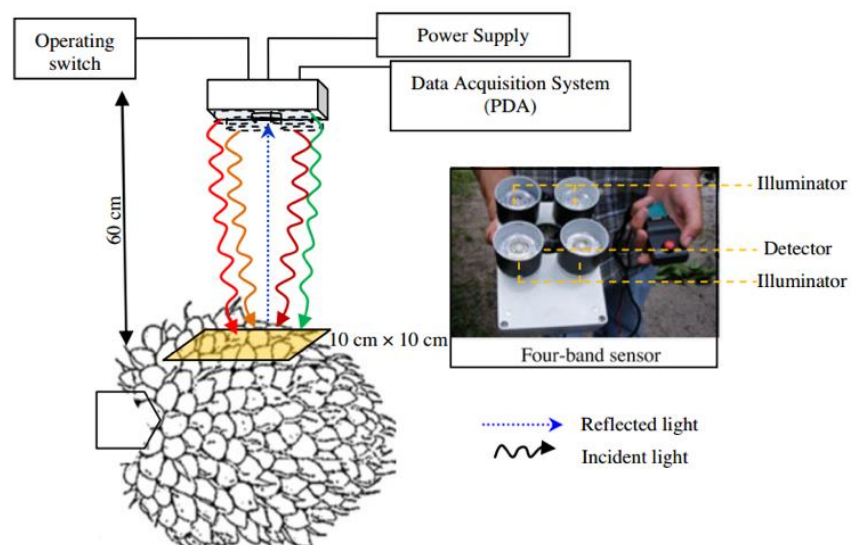

Figure 8. Experimental set-up of four-band optical sensor system (Saeed et al., 2012)

(linear, quadratic, Mahalanobis distance), classification and regression tree, soft independent modelling of classification analogies (SIMCA) and k-nearest neighbour (kNN) were the classification algorithms that were tested in the study. Among the classification algorithms, the quadratic discriminant analysis and discriminant analysis with Mahalanobis distance classifiers attained highest average overall accuracies of above $85 \%$ in FFB maturity classification. Therefore, the portable four-band optical sensor system was applicable to classify the maturity of oil palm FFB.

Among the non-destructive techniques discussed in this section, most of the techniques are applied to detect the ripeness level of oil palm FFB because the maturity of FFB has a direct impact on the quality of extracted oil that will eventually affect the economic value of palm oil.

\section{Future trend}

Measuring the quality of oil palm fresh fruit bunches is an essential step before harvesting in order to ensure maximum oil extraction while minimizing expenses on labor, transportation and processing. To date, a lot of researches have been conducted in developing non-destructive grading systems for evaluating oil palm FFB. There are simple grading systems but also sophisticated systems that relate many quality parameters. Both systems have been proven to have the abilities in achieving high successful rate and accuracy. Commercialization of these nondestructive grading systems is necessary so that the purpose of developing such systems could be met, that is to introduce advanced technologies into oil palm agricultural sector. As mentioned in Mohd Ali et al. (2017), the success of non- destructive techniques relies on the generalization of the hardware system to deliver user-friendly and affordable nondestructive equipment. Thus, a cost-effective FFB classification system that could be operated easily should be implemented. Since the current developed systems consist of image acquisition and data processing, unexpected troubleshooting problems might occur during operation. In order to enhance the application of the generated system, these problems should be addressed in an effective way through developing orientated algorithms that can perform fast problem solving in real-time situation. Additionally, this can also lower the maintenance cost. An automated grading system that is environmentally friendly would be an extra plus point to the respective system. Lastly, further development of the available non-destructive techniques should be taken into action in order to maximize the practical usage while contributing to the society especially the oil palm sector in Malaysia.

\section{Conclusion}

This review has covered the approach of non-destructive techniques in measuring the quality of oil palm fresh fruit bunches. A few applications of non-destructive techniques are by using machine vision inspection system, VIS/NIR spectroscopy, relative entropy-based image processing, fluorescence technique, Kinect camera, and portable four-band optical sensor system. The main quality parameter that is being evaluated is the ripeness level of the FFB. Besides maturity level detection, machine vision inspection system and VIS/NIR spectroscopy are capable to determine the oil content and free fatty acid in FFB whereas the Kinect camera is able to find out the volume of FFB. Concisely, the application of non-destructive techniques is beneficial and impactful to the development of oil palm sector.

\section{Author contributions}

Chen Yee Being carried out the literature review and performed the main writing part. Norhashila Hashim supported the study by providing the concept and structure of the manuscript and supervised the study as well as giving her advice on the manuscript. Bernard Maringgal and Mohd Hafizz Wondi edited the final manuscript.

\section{Conflict of interests}

The authors declare that they have no competing interests to disclose that might be perceived as affecting the objectivity of this mini review.

\section{Acknowledgments}

The authors are thankful to Department of Biological and Agricultural Engineering, Universiti Putra Malaysia for providing facilities in this study.

\section{References}

Harun, N. H., Misron, N., Sidek, R. M., Aris, I., Ahmad, D., Wakiwaka, H., \& Tashiro, K. (2013). Investigations on a novel inductive concept frequency technique for the grading of oil palm fresh fruit bunches. Sensors, 13, 22542266. https://doi.org/10.3390/s130202254

Hazir, M. H. M., Shariff, A. R. M., \& Amiruddin, M. D. (2012). Determination of oil palm fresh fruit bunch ripeness - based on flavonoids and anthocyanin content. Industrial Crops and Products, 36, 466-475. https://doi.org/10.1016/j.indcrop.2011.10.020 
Hazir, M. H. M., Shariff, A. R. M., Amiruddin, M. D., Ramli, A. R., \& Iqbal Saripan, M. (2012). Oil palm bunch ripeness classification using fluorescence technique. Journal of Food Engineering, 113, 534-540. https://doi.org/10.1016/j.jfoodeng.2012.07.008

Kassim, M. S. M., Ismail, W. I. W., Ramli, A. R., \& Bejo, S. K. (2014). Image clustering technique in oil palm fresh fruit bunch (FFB) growth modeling. Agriculture and Agricultural Science Procedia, 2, 337-344. https://doi.org/10.1016/j.aaspro.2014.11.047

Kushairi, A., Ong-Abdullah, M., Nambiappan, B., Hishamuddin, E., Bidin, M. N. I. Z., Ghazali, R., Parveez, G. K. A. (2019). Oil palm economic performance in Malaysia and R\&D progress in 2018. Journal of Oil Palm Research, 31, 165-194. https://doi.org/10.21894/jopr.2019.0026

Makky, M. (2016). A portable low-cost non-destructive ripeness inspection for oil palm FFB. Agriculture and Agricultural Science Procedia, 9, 230-240. https://doi.org/10.1016/j.aaspro.2016.02.139

Makky, M., \& Soni, P. (2014). In situ quality assessment of intact oil palm fresh fruit bunches using rapid portable noncontact and non-destructive approach. Journal of Food Engineering, 120, 248-259. https://doi.org/10.1016/j.jfoodeng.2013.08.011

Mohd Ali, M., Hashim, N., \& Hamid, A. S. A. (2020). Combination of laser-light backscattering imaging and computer vision for rapid determination of oil palm fresh fruit bunches maturity. Computer and Electronic in Agriculture, 169, 105235. https://doi.org/10.1016/j.compag.2020.105235

Mohd Ali, M., Hashim, N., Bejo, S. K., \& Shamsudin, R. (2017). Rapid and nondestructive techniques for internal and external quality evaluation of watermelons: A review. Scientia Horticulturae, 225, 689-699. https://doi.org/10.1016/j.scienta.2017.08.012

Pamornnak, B., Limsiroratana, S., Khaorapapong, T., Chongcheawchamnan, M., \& Ruckelshausen, A. (2017). An automatic and rapid system for grading palm bunch using a Kinect camera. Computers and Electronics in Agriculture, 143, 227-237. https://doi.org/10.1016/j.compag.2017.10.020

Saeed, O. M. Ben, Sankaran, S., Shariff, A. R. M., Shafri, H. Z. M., Ehsani, R., Alfatni, M. S., \& Hazir, M. H. M. (2012). Classification of oil palm fresh fruit bunches based on their maturity using portable four-band sensor system. Computers and Electronics in Agriculture, 82, 55-60. https://doi.org/10.1016/j.compag.2011.12.010

Taparugssanagorn, A., Siwamogsatham, S., \& Pomalaza-Ráez, C. (2015). A non-destructive oil palm ripeness recognition system using relative entropy. Computers and Electronics in Agriculture, 118, 340-349. https://doi.org/10.1016/j.compag.2015.09.018 